\title{
A Distributed Real-Time Tele-operation System Based on the TMO Modeling*
}

\author{
Hanku Lee ${ }^{1}$ and Segil Jeon ${ }^{2}$ ** \\ ${ }^{1}$ School of Internet and Multimedia Engineering, Konkuk University, Seoul, Korea \\ hlee@konkuk.ac.kr \\ ${ }^{2}$ BioMolecular Informatics Center, Konkuk University, Seoul, Korea \\ Tel.: +82-11-9154-3793 \\ sgjeonaricl.konkuk.ac.kr
}

\begin{abstract}
The fast development of grid computing environments makes it possible to access geographically distributed remote instruments, experimental equipments, databases, human resources with respect to real-time in grid computing environments. With conventional programming method, it is very difficult to implement real-time models in uncontrolled distributed environments and to support well-defined interfaces from real-time systems to external systems. We propose an easy-to-use TMO-based tele-operation model with less strict real-time constraints in grid environments. Using the proposed model, we design and develop a TMO-based tele-operation system for real industrial applications used for tsunami-detecting instruments.
\end{abstract}

\section{Introduction}

Today we access geographically distributed remote instruments, experimental equipments, databases, human resources, high-performance computers, etc, as if accessing local resources from a long distance away. But it brings us another side: How are these instruments, devices and data well-synchronized in distributed real-time systems? With conventional programming methods it is very difficult to design and implement well-defined real-time models in uncontrolled distributed environments.

We propose a TMO-based distributed real-time tele-operation model with less strict real-time constraints in grid environments. The proposed model can be used to control tsunami-detecting instruments. For example, a remote meteorologist can control and monitor tsunami-detecting instruments and conference with local meteorologists from a long distance away using the proposed model in grid computing environments.

In the next section, we discuss related works such as TMO, Distributed Objectoriented Freeway Simulator (DOFS), Real-time CORBA. Then, we propose a TMObased tele-operation model and mention design and implementation issues in section 3. Section 4 concludes.

\footnotetext{
* This paper was supported by Konkuk University in 2006 .
}

** Corresponding author. 


\section{Related Works}

The Time-Triggered Message-Triggered Object (TMO) was established in early 1990's with a concrete syntactic structure and execution semantics for economical reliable design and implementation of RT systems $[1,2,4,5]$. TMO is a high-level real-time computing object. It is built in standard C++ and APIs called TMO Support Library (TMOSL).

TMO contains two types of methods, time-triggered methods (SpM), which are clearly separated from the conventional service methods (SvM). The SpM executions are triggered upon reaching of the RT clock at specific values determined at the design time whereas the SvM executions are triggered by service request messages from clients. Moreover, actions to be taken at real times which can be determined at the design time can appear only in SpM's. Real-time Multicast and Memory Replication Channel (RMMC) is an alternative to the remote method invocation for facilitating interactions among TMOs. Use of RMMCs tends to lead to better efficiency than the use of traditional remote method invocations does in the area of distributed multimedia applications that involve frequent delivery of the same data to more than two participants distributed among multiple nodes.

Distributed Object-oriented Freeway Simulator (DOFS) [3] is a freeway automobile traffic simulator conducting with the goal of validating the potential of the TMO structuring scheme supported by the recently implemented TMOSM. DOFS is intended to support serious studies of advanced freeway management systems by providing high-resolution high-accuracy easily expandable freeway simulation. The system can help the Driver avoiding the traffic road and supply real-time traffic information. The TMO scheme brings major improvement in the RT system design and implementation efficiency.

The Real-time CORBA (RT-CORBA) [6] is an optional set of extensions to CORBA to be used as a component of a real-time system. It is designed for applications with hard real-time requirements, such as avionics mission computing, as well as those stringent soft real-time requirements, such as telecommunication call processing. Moreover, to allow applications to control the underlying communication protocols and end-system resources, the Real-time CORBA specification defines standard interfaces that can be used to select and configure certain protocol properties.

\section{The Proposed TMO-Based Tele-operation Model}

\subsection{Architecture}

Due to the fast development of the Internet and grid computing environments, it is possible for engineers and researchers to access remote instruments and computing resources from a long distance away. However, we need to support real-time controls and the timing characteristics on these geographically distributed, grid-enabled, and real-time applications without pain during the development. 


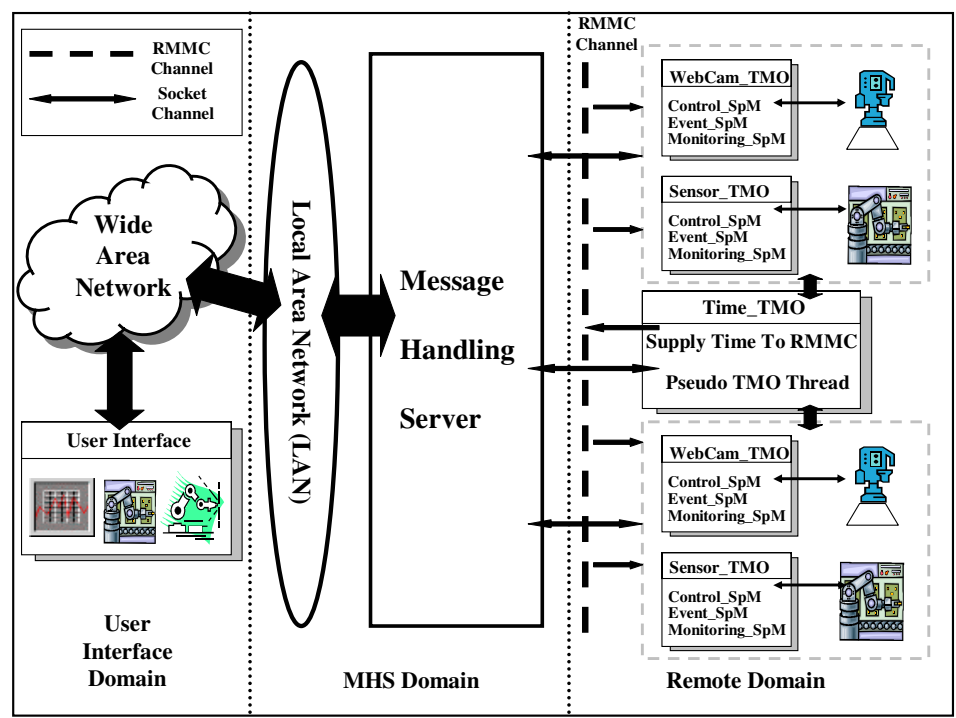

Fig. 1. The Architecture of TMO-Based Tele-Operation Model

Figure 1 depicts the architecture of the proposed TMO-based tele-operation model. One of the main issues for the proposed model is to apply the easy-to-use TMO to real-time applications that are usually hard to design and implement with conventional programming methods. The proposed model is divided to 3 domains: remote domain, message-handling-service domain, user interface domain.

The remote domain (RD) is to collect remote data and to monitor remote instruments. RD consists of the Time TMO and working TMOs. The Time TMO gives the timing characteristics to other working TMOs (e.g. WebCam_TMO and Sensor_TMO) via the Real-time Multicast and Memory Replication Channel (RMMC). The video, audio, and sensor data with the timing characteristics are transferred via the socket channel to the message-handling-service domain The time characteristics supplied by the Time TMO are more suitable to the proposed model than those supplied by the Internet or GPS time services since the Time TMO is closely located to other working TMOs and this locality avoids the network latency that makes it hard to synchronize real-time applications.

The message-handling-service domain (MHSD) is to manage message-handling servers in order to help data communication between UID and RD. MHSD provides the grid-enabled environments based on the TCP/IP-based client/server model and grid applications to handle control-messages between UID and RD to be safely and precisely transferred. MHSD should keep waking up, be started prior to other domains, and wait for control-messages. Servers in MHSD can storage a large amount of data from the remote domain and can provide the secure management of data from the remote domain to the interfaces.

Finally, the user interface domain (UID) is to provide user interfaces to check the status of the whole system, to manage incoming and outgoing control-messages between the client and remote instruments, and to handle real-time information needed for the tele-operation application for the client. This domain is implemented in MFC. 


\subsection{Implementation}

In this section we mention several implementation issues for the remote domain in detail. Figure 2 represents the basic structure of the remote domain, called TMObased real-time agent framework for the proposed TMO-based tele-operation system. The real-time agent framework is implemented using TMO toolkit [1]. It consists of the Time TMO and working TMOs (e.g. WebCam_TMO and Sensor_TMO). Moreover, it is basically divided into three services: control-message waiting and processing service, time service, and real-time data processing service.

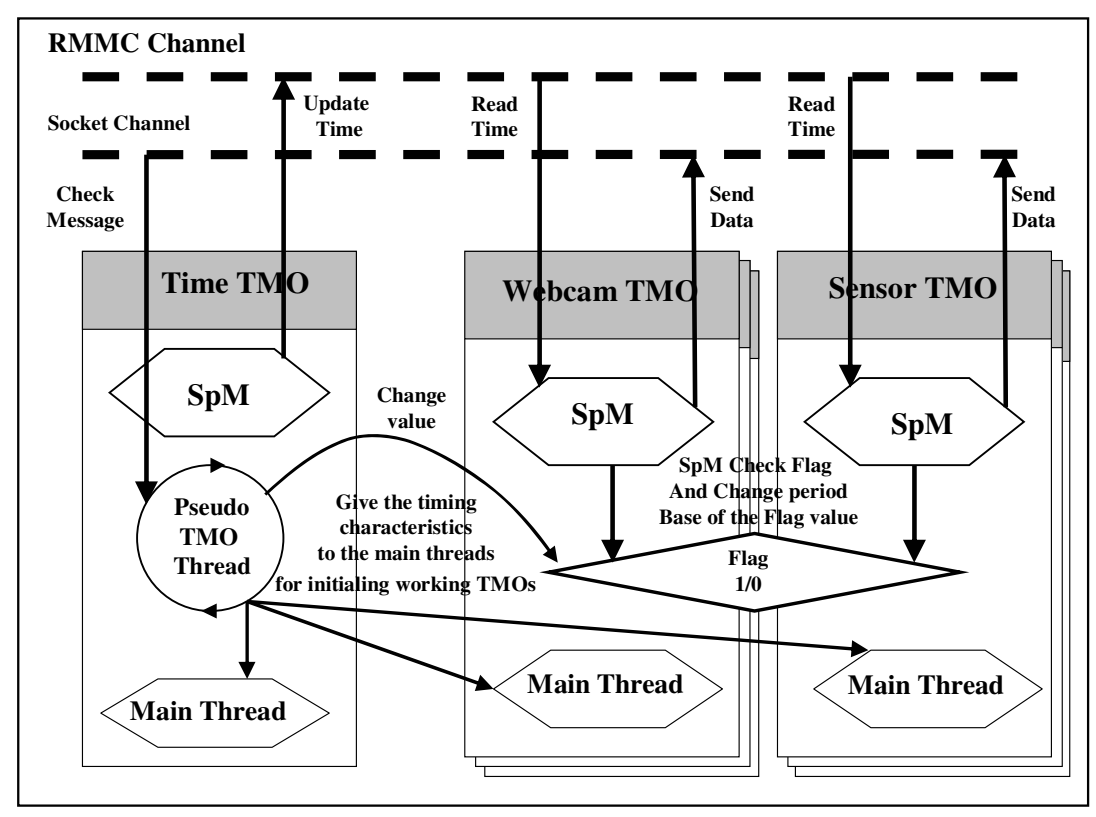

Fig. 2. The Remote Domain: TMO-Based Real-Time Agent framework

The control-message waiting and processing service waits for and processes control-messages from UID according to message types using the Pseudo-TMO-Thread. The Pseudo-TMO-Thread is located in the Time TMO and gives the timing characteristics to the main threads for initialing all working TMOs in the remote domain. But The Pseudo-TMO-Thread keeps waking up unlike SpM of TMO periodically wakes up. Major initialization steps for the agent framework are as follows:

1. The Pseudo-TMO-Thread is invoked and checks up the header information of control-messages from MHSD.

2. Each message is classified such as WebCamMessage, and is sent to its designated TMO.

3. Each TMO extracts the timing characteristics from its control-message, initializes its time, and builds up the socket channel among other TMOs. 
4. The TMO middleware is activated.

5. The TMO-based real-time agent framework is activated.

The Pseudo-TMO-Thread keeps waking up and getting control-messages from UID. If a control-message is a normal command to control remote instruments, then the Pseudo-TMO-Thread does normal real-time data processing in this service. But if a control-message is a command to scale up or down the whole system in the time dimension, then the Pseudo-TMO-Thread extracts data out of the message, stops the designated SpM for a while, changes the period of the designated SpM, and restarts the designated SpM. When a working TMO needs to process video data, sometimes, the data processing time exceeds the period of SpM for the TMO. It happens because of the network latency or the size of the video data. In this case, the period of SpM should be extended more than the data processing time.

For example, when the network becomes delayed, the data transfer from web cameras becomes delayed as well. To avoid the latency of the whole system because of the latency of the video transfer, the Pseudo-TMO-Thread gets a control-message from UID to change the period of SpM for web cameras from 1 second to 3 seconds. After the network becomes normal, the Pseudo-TMO-Thread gets another controlmessage from UID to change the period back. This functionality makes the TMObased real-time agent framework flexible for real-time monitoring.

The time service is served by the Time TMO that is closely located to other working TMOs. The time service synchronizes the timing characteristics of each SpM in working TMOs. The real-time data processing service manages data processing according to the timing characteristics of each SpM and attaches the timing characteristics on video and sensor data. The time service and the real-time data processing service use RMMC that is a real-time communication channel among working TMOs to broadcast common information such as the timing characteristics and memory for working TMOs. RMMC is a good alternative to the Internet or GPS time services since it avoids the network latency that makes it hard to synchronize real-time applications. SpM of the Time TMO periodically (e.g. 200 micro-seconds) updates the timing characteristics of RMMC using its own timing characteristics. Then each SpM reads the timing characteristics of RMMC, attaches it on video, audio, and sensor data, and transfer data to MHSD.

Figure 3, 4, and 5 represents a distributed, real-time tele-operation system to detect tsunami based on our proposed model. The TMO-based tele-operation system is a real-time, tele-operation, and tele-monitoring system. Using the tsunami detecting system, a meteorologist can monitor the ocean, control the instruments, and conference with local engineers from a long distance away. In detail, first, a remote meteorologist monitors the current status of a designated point of the ocean on his/her computer from a long distance away. Second, the video, audio, and sensor data from the point are collected and synchronized by the TMO-based real-time agent framework and transferred via MHSD to the remote engineer. Third, the data are updated and displayed on his/her computer. Finally, the remote meteorologist can control remote instruments by controllers on his/her computer, send control- 
messages such as scaling up or down the whole system in the time dimension, and in advance warn local meteorologists to prepare the natural disaster. Using the system, moreover, the remote meteorologist can chat and talk with local meteorologists.

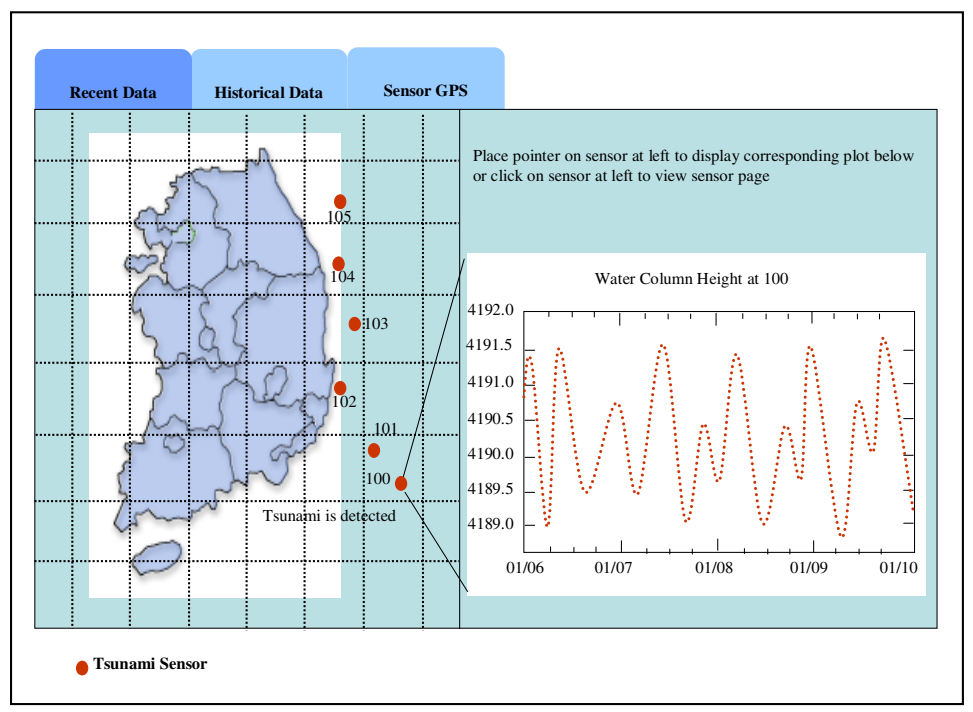

Fig. 3. Historical Data of Tsunami Detecting Tele-Operation System

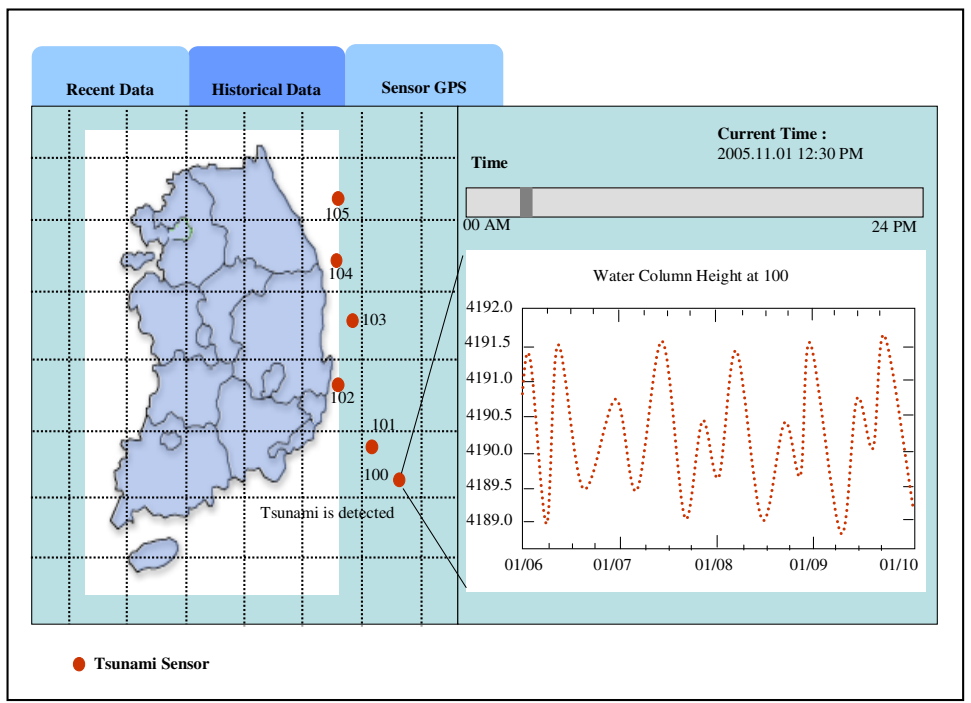

Fig. 4. Historical Data of Tsunami Detecting Tele-Operation System 


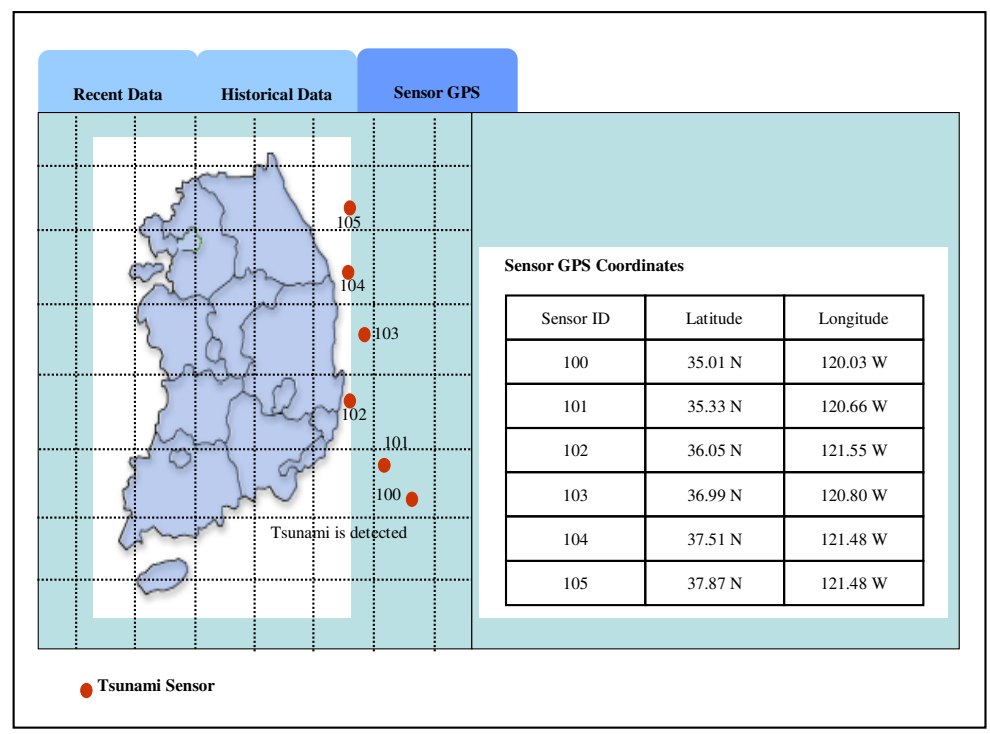

Fig. 5. Sensor GPS Data of Tsunami Detecting Tele-Operation System

\subsection{Advantages and Restrictions of the Proposed Model}

We experienced several advantages of adapting TMO on the proposed model during implementing a TMO-based tele-operation system.

Developers can highly predict the timing performance using TMO during designing and developing the proposed TMO-based model. Execution of time consuming unpredictable I/O operations such as video outputs, keyboard inputs, etc, can be handled by the Pseudo-TMO-Thread. Each TMO thread designated to instruments are not burdened with these suffering tasks.

Moreover, it is easy to implement and debug TMO nodes. Implementing and debugging of real-time controls and the timing characteristics cause pain during the development of distributed real-time applications with conventional real-time methods. But all we need to do is to use communication APIs, thread initializing, managing, and terminating APIs, supported by the TMO tool kit.

It is easy to modify and expand the proposed TMO-based model. We often need to scale up or down the whole system in the time dimension. Many modifications could be needed with conventional real-time methods. But all we need to do is to change the scale of the real-time clock of TMO for the proposed TMO-based model.

We experienced some restrictions that TMO-based real-time applications are not suitable to real-time systems handling huge amount of data in a relatively short SpM wakeup period. For example, wind channel experiments in aerospace researches generally need video capturing instruments taking approximately 1,000 photos per second and the size of each photo is approximately $1 \mathrm{M}$ bytes. In this case, we can easily scale down the period of SpM (e.g. 10 micro-seconds). But it is impossible to process this amount of video data in time with contemporary hardware and network environments. 
Thus, we urge TMO-based real-time applications are suitable to systems with less strict real-time constraints such as tsunami-detecting instruments, etc, since those instruments product relatively small amount of data in the period of SpM and are not a time-critical decision model.

\section{Conclusion}

We proposed an easy-to-use TMO-based tele-operation model with less strict realtime constraints in grid environments. Using the proposed model, we designed and developed a TMO-based tele-operation system for real industrial applications able to be used to control and monitor tsunami-detecting instruments.

The TMO-based tele-operation model, proposed in this paper, is promising since it provides a sound TMO-based real-time agent framework, cost-effectively resolving the problems caused by conventional programming methods during the development. However, the experimental research and development with the proposed model is at an early stage. Moreover, much more research efforts are needed to develop more stable TMO-based real-time agent framework.

We will adapt the proposed model to develop a tsunami detecting system in the future research.

\section{References}

1. TMOSL_v4.0_manual_draft http://dream.eng.uci.edu/TMOdownload/

2. Kim,K.H, "APIs for Real-Time Distributed Object Programming”, IEEE Computer,June 2000,pp.72-80

3. K.H.(Kane) Kim, Juqiang Liu, Masaki Ishida and Inho Kim.: "Distributed Object-Oriented Real-Time Simulation of Ground Transportation Networks with the TMO Structuring Scheme" , Proc. COMPSAC '99 (IEEE CS Computer Software \& Applications Conf.), Phoenix, AZ, Oct. 1999, pp.130-138.

4. Kim, K.H., "Real-Time Object-Oriented Distributed Software Engineering and the TMO Scheme", Int'1 Jour. of Software Engineering \& Knowledge Engineering, Vol. No.2, April 1999, pp.251-276.

5. Kim, K.H., "Object Structures for Real-Time Systems and Simulators", IEEE Computer, August 1997, pp.62-70.

6. Douglas Schmidt, Fred Kuhns, "An overview of the Real-time CORBA Specification", IEEE Computer special issue on Object-Oriented Real-time Distributed Computing, June 2000, pp.56-63.

7. Real-Time for Java Experts Group, "Real-time Specification for Java, Version 0.9.2," 29 Mar. 2000, http://www.rtj.org/public. 